\title{
Emissões otoacústicas em lactentes expostos a infecção intra-útero
}

\section{Otoacoustic emissions in infants exposed to intrauterine infection}

\author{
Jordana Rodrigues da Silveira ${ }^{1}$, Alessandra Spada Durante ${ }^{2}$, Kátia de Almeida ${ }^{3}$, \\ Carlos Kazuo Taguchi ${ }^{4}$, Maria Cecília Greco ${ }^{5}$
}

\begin{abstract}
RESUMO
Objetivo: Analisar as Emissões Otoacústicas (EOA) em lactentes expostos à infecção intra-útero. Métodos: Foi realizada a captação das EOA por transiente (EOAT) e das EOA produto de distorção (EOAPD) em 40 lactentes: 14 lactentes portadores e/ou expostos a algum tipo de infecção intra-útero (Grupo Estudo) e 26 lactentes sem intercorrências e sem indicadores de risco auditivo (Grupo Comparação). Os critérios de inclusão foram: Grupo Comparação: lactentes nascidos a termo, saudáveis e sem indicador de risco para alteração auditiva; Grupo Estudo: lactentes nascidos a termo, ou com idade gestacional corrigida entre 37 e 41 semanas na data de avaliação e portador ou exposto a algum tipo de infecção intra-útero. A análise estatística do conjunto de dados foi efetuada utilizando os testes estatísticos não paramétricos de Wilcoxon e Mann-Whitney, e a técnica de intervalo de confiança para média. Resultados: Foram observados menores níveis de resposta e relação sinal/ruído das EOAT e EOAPD no Grupo Estudo em relação ao Grupo Comparação, com valores estaticamente significantes para as EOAPD em $8000 \mathrm{~Hz}$ e para EOAT em 1,5 Hz. Conclusão: A exposição à infecção intra-útero pode atenuar o nível de resposta das emissões otoacústicas no período neonatal.
\end{abstract}

Descritores: Emissões otoacústicas espontâneas; Lactente; Doenças fetais; Infecção/congênito; Fatores de risco

\section{INTRODUÇÃO}

A audição é o meio pelo qual o recém-nascido entra em contato com o mundo sonoro e com as estruturas da língua oral ${ }^{(1)}$.

Nos últimos anos vem se intensificando o interesse em estudos sobre a importância da triagem auditiva neonatal. A prevenção, detecção e a possibilidade de potencializar o desenvolvimento de neonatos com alteração auditiva vêm sendo muito discutidas.

Pesquisa desenvolvida no Curso de Fonoaudiologia da Faculdade de Ciências Médicas da Santa Casa de São Paulo - FCMSCSP - São Paulo (SP), Brasil.

(1) Especialista em Audiologia; Fonoaudióloga junto à Santa Casa de Misericórdia de Santo Amaro - São Paulo (SP), Brasil.

(2) Doutora, Professora Assistente do Curso de Graduação em Fonoaudiologia da Faculdade de Ciências Médicas da Santa Casa de São Paulo - FCMSCSP - São Paulo (SP), Brasil.

(3) Doutora, Professora Adjunto do Curso de Graduação em Fonoaudiologia da Faculdade de Ciências Médicas da Santa Casa de São Paulo - FCMSCSP - São Paulo (SP), Brasil.

(4) Doutor, Professor Adjunto da Universidade Federal de Sergipe - UFS São Cristóvão (SE), Brasil.

(5) Mestre, Professora instrutora do Curso de Graduação em Fonoaudiologia da Faculdade de Ciências Médicas da Santa Casa de São Paulo - FCMSCSP - São Paulo (SP), Brasil.

Endereço para correspondência: Jordana Rodrigues da Silveira. R. Irineu Fernandes de Souza, 166, Jardim Maristela, Ribeirão Pires (SP), Brasil, CEP: 09423-050. E-mail: jordana.rodrigues@ gmail.com

Recebido em: 2/3/2009; Aceito em: 12/10/2009
Estudos $^{(2,3)}$ mostram que se faz cada vez mais necessária a realização do monitoramento auditivo logo no começo da infância, sendo que em cada 1000 crianças 20 sofrem alterações na audição até completar sete anos de idade.

As doenças infecto-contagiosas, as afecções de orelha média e problemas progressivos de origem genética ou congênita são as causas mais comuns dos distúrbios da audição. Em contrapartida as explicações para cada caso variam conforme o momento em que a perda auditiva se manifesta ${ }^{(3)}$.

Em estudo ${ }^{(4)}$ que caracterizou 360 prontuários de recémnascidos (RNs) de alto risco da Irmandade Santa Casa de Misericórdia de São Paulo (ISCMSP) no Programa de Triagem Auditiva Neonatal (PTAN) foi encontrada a seguinte ocorrência dos indicadores de risco para deficiência auditiva: uso de ototóxicos em 65 neonatos $(25,1 \%)$ e exposição a infecções congênitas em 54 neonatos (21,3\%).

O registro das Emissões Otoacústicas (EOA) tem sido largamente indicado, por ser um exame rápido, de fácil aplicação e podendo atingir um grande número de sujeitos em curto espaço de tempo, portanto útil nos programas de triagem auditiva neonatal.

As EOA foram definidas como a liberação de energia sonora da cóclea, a qual se propaga na orelha média até chegar ao meato acústico externo. Estão presentes em cócleas funcionalmente normais e deixam de ser observadas nos indivíduos com limiares acima de $20-30 \mathrm{dBNA}^{(5)}$. 
As emissões otoacústicas por transiente (EOAT) são provocadas por um sinal transiente ou clique. Uma sonda é usada para apresentar o clique e para registrar a resposta. A sonda é mantida em posição dentro do meato acústico externo por uma oliva flexível. Uma série de estímulos-cliques são apresentados, em geral, em um nível de intensidade entre 80-85 dBNPS pico equivalente. As respostas são detectadas por um microfone dentro da sonda e enviadas para um computador onde o sinal é analisado em uma janela de 12 a 20 milissegundos.

As emissões otoacústicas produto-distorção (EOAPD) ocorrem como resultado de um processo não linear da cóclea. Quando dois tons são apresentados à cóclea, a distorção ocorre na forma de outros tons que não estavam presentes nos dois tons dos sinais estimuladores. Estas distorções são combinações de tons, harmônicos, que estão relacionados aos tons estimuladores de uma forma matemática previsível. Os dois tons usados para provocar as EOAPD são, por convenção, denominados f1 e f2. O produto de distorção mais robusto, ou intenso, ocorre na frequência representada pela equação $2 \mathrm{f} 1$ - f2.

Embora exista uma maior atenção voltada para a detecção precoce de alterações auditivas, são poucos os trabalhos disponíveis, na literatura, que caracterizam as EOA em neonatos com indicadores de risco auditivo.

Vislumbrando aprimorar o potencial diagnóstico das emissões otoacústicas, primordialmente direcionadas aos programas de triagem auditiva, as características das EOAT e das EOAPD foram investigadas pelo presente estudo na população neonatal.

Com a hipótese de que os lactentes expostos a infecção intra-útero poderiam apresentar função coclear reduzida e consequentemente EOA com menores níveis de resposta, o presente estudo teve como objetivo investigar este grupo, buscando aprimorar a pesquisa diagnóstica e permitir a identificação precoce de alterações auditivas.

\section{MÉTODOS}

O projeto, bem como o Termo de Consentimento Livre e Esclarecido, foi aprovado pelo Comitê de Ética em Pesquisa da Irmandade Santa Casa de Misericórdia de São Paulo processo no 194/06.

Foi realizado um estudo de caráter exploratório, prospectivo, analítico e transversal e foram avaliados um total de 40 lactentes do alojamento conjunto e do berçário da Irmandade da Santa Casa de Misericórdia de São Paulo, no período de fevereiro a junho de 2007. Os exames foram realizados na Clínica de Fonoaudiologia da Faculdade de Ciências Médicas da Santa Casa de São Paulo. Os lactentes foram divididos em dois grupos:

- Grupo Comparação: composto de 26 lactentes, sendo 15 do gênero feminino e 11 do gênero masculino, todos nascidos a termo, saudáveis e sem indicador de risco para alteração auditiva e que apresentaram EOAT e EOAPD presentes em ambas as orelhas.

- Grupo Estudo: Composto de 14 lactentes, sendo seis do gênero feminino e oito do gênero masculino, nascidos a termo, ou com idade gestacional corrigida entre 37 e 41 semanas na data de avaliação e portador ou exposto a algum tipo de infecção congênita.

\section{Equipamento}

A medida das EOA foi realizada por meio de analisador de emissões cocleares Capella-Madsen (GN Otometrics ${ }^{\circledR}$ ) que estava acoplado a um computador Dell® com monitor colorido de 17 polegadas e processador Celleron $®$, funcionando com o Microsoft Windows ${ }^{\circledR}$ junto com a plataforma do software NOAH.

Para o registro das EOAT utilizou-se o modo não linear, intensidade 75-80 dB pico equivalente duração de $40 \mu$ s; polaridade condensada; estímulos de $40 \mathrm{~ms}$; janela de 3 a $12,5 \mathrm{~ms}$ nas frequências de 1,$0 ; 1,5 ; 2,0 ; 3,0 ; 4,0 \mathrm{kHz}$ e geral e foram coletados 2080 registros.

Para o registro das EOAPD utilizou-se os seguintes parâmetros: $\mathrm{L}_{1}=55 \mathrm{~dB}$ e $\mathrm{L}_{2}=65 \mathrm{~dB} ; \mathrm{f}_{1} / \mathrm{f}_{2}=1,2 ; \mathrm{PD}=2 \mathrm{f}_{1}-\mathrm{f}_{2}$; tempo de 100 segundos; frequências testadas $=1,5 ; 2,0 ; 3,0 ; 4,0 ; 6,0$; $8,0 \mathrm{kHz}$ e até 1000 registros coletados ou relação sinal/ruído $(\mathrm{S} / \mathrm{R})=6 \mathrm{~dB}$.

\section{Procedimento}

O registro das EOAT e EOAPD foi realizado com o lactente tranquilo, sem atividade corporal. As EOAT e EOAPD foram registrados em ambas as orelhas.

Para cada unidade amostral foram registradas as seguintes variáveis:

- EOAT: nível de resposta e relação S/R por bandas de frequência $(1 ; 1,5 ; 2 ; 3$ e 4 kHz) e geral.

- EOAPD: nível de resposta e relação S/R das frequências 1,5;

$2 ; 3 ; 4 ; 6$ e $8 \mathrm{kHz}$.

\section{Análise estatística}

Para este trabalho utilizou-se os testes estatísticos não paramétricos de Wilcoxon e Mann-Whitney. Na complementação da análise descritiva, fez-se uso da técnica de intervalo de confiança para média.

\section{RESULTADOS}

O Grupo Comparação (GC) (N=26) e o Grupo Estudo (GE) $(\mathrm{N}=14)$ apresentaram características perinatais semelhantes. As medidas de tendência central da idade gestacional (em semanas) e do peso ao nascimento foram discretamente menores no grupo estudo. Os lactentes que compuseram o grupo estudo apresentaram o indicador de risco auditivo infecções congênitas $(100 \%)$ e apenas um lactente $(7,14 \%)$ apresentou a associação do uso de ototóxicos. A sífilis foi a infecção intra-útero mais encontrada no grupo estudo (43\%), seguida do HIV $(28,57 \%)$ e toxoplasmose $(26,66 \%)$. Além disso, um lactente apresentou citomegalovírus $(7,14 \%)$ associado com a toxoplasmose.

Na análise do nível de resposta e da relação S/R das EOAT, o GC apresentou maiores valores na orelha direita (OD) do que na orelha esquerda (OE), com significância estatística apenas para a banda de frequência de $1 \mathrm{kHz}$ (Tabela 1).

Para o GE tanto o nível de respostas quanto a relação $S / R$ das EOAT apresentaram maiores valores na OD do que na $\mathrm{OE}$, 
Tabela 1. Nível de resposta e relação S/R das EOAT geral e por banda de frequência para GC segundo orelha e gênero

\begin{tabular}{|c|c|c|c|c|c|c|c|c|c|}
\hline EOAT & Frequência & Orelha & Média & IC & Valor de $p$ & Gênero & Média & IC & Valor de $p$ \\
\hline \multirow{12}{*}{$\begin{array}{l}\text { Nível de } \\
\text { resposta } \\
\text { (dB NPS) }\end{array}$} & \multirow[t]{2}{*}{$1 \mathrm{kHz}$} & OD & 14,80 & 1,56 & \multirow[t]{2}{*}{$0,041^{*}$} & Fem & 15,58 & 1,72 & \multirow[t]{2}{*}{$0,003^{*}$} \\
\hline & & OE & 12,02 & 2,18 & & Masc & 11,03 & 1,80 & \\
\hline & \multirow[t]{2}{*}{$1,5 \mathrm{kHz}$} & OD & 16,23 & 2,37 & \multirow[t]{2}{*}{0,983} & Fem & 17,40 & 1,78 & \multirow[t]{2}{*}{ 0,070\# } \\
\hline & & $\mathrm{OE}$ & 15,45 & 2,02 & & Masc & 14,14 & 2,51 & \\
\hline & \multirow[t]{2}{*}{$2 \mathrm{kHz}$} & OD & 19,23 & 1,87 & \multirow[t]{2}{*}{0,240} & Fem & 19,01 & 1,80 & \multirow[t]{2}{*}{0,452} \\
\hline & & OE & 17,46 & 1,59 & & Masc & 17,62 & 1,65 & \\
\hline & \multirow[t]{2}{*}{$3 \mathrm{kHz}$} & OD & 22,33 & 2,30 & \multirow[t]{2}{*}{0,983} & Fem & 22,55 & 2,15 & \multirow[t]{2}{*}{0,411} \\
\hline & & OE & 21,26 & 1,73 & & Masc & 20,97 & 1,79 & \\
\hline & \multirow[t]{2}{*}{$4 \mathrm{kHz}$} & OD & 18,91 & 2,41 & \multirow[t]{2}{*}{0,777} & Fem & 19,89 & 2,25 & \multirow[t]{2}{*}{0,280} \\
\hline & & OE & 18,68 & 2,21 & & Masc & 17,60 & 2,28 & \\
\hline & \multirow[t]{2}{*}{ Geral } & OD & 27,45 & 1,79 & \multirow[t]{2}{*}{0,408} & Fem & 27,81 & 1,69 & \multirow[t]{2}{*}{0,244} \\
\hline & & OE & 26,44 & 1,37 & & Masc & 25,99 & 1,34 & \\
\hline \multirow{12}{*}{$\begin{array}{l}\text { Relação S/R } \\
\text { (dB NPS) }\end{array}$} & \multirow[t]{2}{*}{$1 \mathrm{kHz}$} & OD & 9,08 & 3,37 & \multirow[t]{2}{*}{$0,039^{*}$} & Fem & 7,61 & 3,18 & \multirow[t]{2}{*}{0,934} \\
\hline & & OE & 4,55 & 2,08 & & Masc & 5,95 & 2,46 & \\
\hline & \multirow[t]{2}{*}{$1,5 \mathrm{kHz}$} & OD & 16,07 & 3,21 & \multirow[t]{2}{*}{0,396} & Fem & 17,35 & 3,19 & \multirow[t]{2}{*}{0,431} \\
\hline & & OE & 16,10 & 2,99 & & Masc & 14,70 & 2,82 & \\
\hline & \multirow[t]{2}{*}{$2 \mathrm{kHz}$} & OD & 19,91 & 2,50 & \multirow[t]{2}{*}{0,469} & Fem & 18,16 & 2,09 & \multirow[t]{2}{*}{0,196} \\
\hline & & OE & 18,14 & 18,95 & & Masc & 19,98 & 2,63 & \\
\hline & \multirow[t]{2}{*}{$3 \mathrm{kHz}$} & OD & 20,57 & 19,55 & \multirow[t]{2}{*}{0,794} & Fem & 20,17 & 2,34 & \multirow[t]{2}{*}{0,630} \\
\hline & & OE & 19,05 & 20,85 & & Masc & 19,42 & 2,04 & \\
\hline & $4 \mathrm{kHz}$ & OD & 16,83 & 15,65 & 0,913 & Fem & 16,90 & 2,21 & 0,385 \\
\hline & & OE & 15,75 & 15,90 & & Masc & 15,62 & 2,61 & \\
\hline & Geral & OD & 15,16 & 15,60 & 0,777 & Fem & 14,25 & 1,92 & 0,664 \\
\hline & & OE & 14,05 & 13,75 & & Masc & 14,99 & 2,17 & \\
\hline
\end{tabular}

*Valores estatisticamente significantes $(p<0,05)$

\# Valores próximos ao nível de significância adotado

Legenda: $E O A T=$ emissões otoacústicas por transientes; $S / R=$ sinal/ruído; OD = orelha direita; OE = orelha esquerda; IC = intervalo de confiança; Fem = feminino; Masc = masculino

exceto no nível de respostas da banda de frequência $2 \mathrm{kHz}$ e na relação $\mathrm{S} / \mathrm{R}$ das bandas de frequências de 1 e $2 \mathrm{kHz}$, porém sem significância estatística (Tabela 2).

Os níveis de resposta e relação sinal ruído das EOAT apresentaram valores maiores para o GC em relação ao GE exceto na relação $\mathrm{S} / \mathrm{R}$ das bandas de frequência de 3 e 4 kHz com valores estatisticamente significantes para o nível de resposta da banda de frequência de $1,5 \mathrm{kHz}$ (Tabela 3).

$\mathrm{Na}$ análise do efeito de grupo as EOAPD apresentaram, em geral, maiores níveis de respostas e relação S/R para o GC, com significância estatística apenas em $8 \mathrm{kHz}(\mathrm{p}=0,009)$ (Tabela 4).

\section{DISCUSSÃO}

O desenvolvimento deste trabalho permitiu o contato com as realidades da região e as dificuldades da população na adesão a programas de saúde. O Grupo Estudo (GE) dessa casuística contou com 14 lactentes (60,8\% de adesão) dos 23 encaminhados para a realização do exame, e 26 lactentes para o Grupo Comparação (GC) $(28,8 \%$ de adesão) dos 90 neonatos encaminhados.

$\mathrm{O}$ índice maior de faltas foi no GC (71,2\%). Apesar das mães concordarem com a participação na pesquisa na fase de alta hospitalar, não foram realizar o exame. Acredita-se que o fato do lactente ser saudável associado às dificuldades acima citadas, acentue a maior porcentagem de abandono no GC, este dado também foi observado em outros estudos ${ }^{(6,7)}$.

De acordo com evidenciado por outros trabalhos ${ }^{(8,9)}$ que, ao discutirem as EOAT em neonatos, apontam que com 38 semanas de idade gestacional corrigida o lactente estaria "fora" do período de imaturidade do sistema auditivo. O presente estudo teve o cuidado de utilizar o parâmetro da idade gestacional corrigida, a fim de que a idade não interferisse no resultado do exame. Dessa forma, os lactentes nascidos prematuros foram avaliados com idade gestacional corrigida entre 37 e 41 semanas na data de avaliação. Para corrigir a idade gestacional, utilizou-se a idade gestacional obtida pelo método de New Balard ${ }^{(10)}$.

Foram excluídos os neonatos com quaisquer indicadores de perda auditiva que não fossem a infecção congênita, com a finalidade de avaliar as características típicas desse fator de risco auditivo. Foi mantido o caso com associação de uso de ototóxico por não haver discrepância dos dados, de acordo com a análise estatística. A ocorrência das infecções congênitas na população estudada foi de $2,88 \%$, bem superior à ocorrência de $1,17 \%$ apontado em estudo espanhol ${ }^{(1)}$ e de $0,3 \%{ }^{(7)}$ destacada 
Tabela 2. Nível de resposta e relação S/R das EOAT geral e por banda de frequência para GE segundo a orelha e o gênero

\begin{tabular}{|c|c|c|c|c|c|c|c|c|c|}
\hline EOAT & Frequência & Orelha & Média & IC & Valor de $p$ & Gênero & Média & IC & Valor de $p$ \\
\hline \multirow{12}{*}{$\begin{array}{l}\text { Nível de } \\
\text { resposta } \\
\text { (dB NPS) }\end{array}$} & \multirow[t]{2}{*}{$1 \mathrm{kHz}$} & OD & 14,64 & 3,02 & \multirow[t]{2}{*}{0,327} & Fem & 13,52 & 4,04 & \multirow[t]{2}{*}{0,979} \\
\hline & & OE & 12,12 & 3,33 & & Masc & 13,11 & 2,69 & \\
\hline & \multirow[t]{2}{*}{$1,5 \mathrm{kHz}$} & OD & 15,09 & 2,49 & \multirow[t]{2}{*}{0,239} & Fem & 14,89 & 3,71 & \multirow[t]{2}{*}{0,436} \\
\hline & & OE & 13,31 & 2,89 & & Masc & 13,57 & 1,96 & \\
\hline & \multirow[t]{2}{*}{$2 \mathrm{kHz}$} & OD & 16,53 & 2,10 & \multirow[t]{2}{*}{0,239} & Fem & 16,65 & 3,34 & \multirow[t]{2}{*}{0,835} \\
\hline & & OE & 17,46 & 3,17 & & Masc & 17,31 & 2,29 & \\
\hline & \multirow[t]{2}{*}{$3 \mathrm{kHz}$} & OD & 20,53 & 3,02 & \multirow[t]{2}{*}{0,695} & Fem & 19,55 & 4,03 & \multirow[t]{2}{*}{0,815} \\
\hline & & OE & 19,07 & 2,72 & & Masc & 19,88 & 1,95 & \\
\hline & \multirow[t]{2}{*}{$4 \mathrm{kHz}$} & OD & 18,03 & 3,05 & \multirow[t]{2}{*}{0,875} & Fem & 18,23 & 3,65 & \multirow[t]{2}{*}{0,959} \\
\hline & & $\mathrm{OE}$ & 18,03 & 2,25 & & Masc & 17,89 & 1,83 & \\
\hline & \multirow[t]{2}{*}{ Geral } & OD & 25,65 & 2,41 & \multirow[t]{2}{*}{0,814} & Fem & 25,67 & 2,94 & \multirow[t]{2}{*}{0,917} \\
\hline & & OE & 25,16 & 2,01 & & Masc & 25,18 & 1,63 & \\
\hline \multirow{12}{*}{$\begin{array}{l}\text { Relação S/R } \\
\text { (dB NPS) }\end{array}$} & \multirow[t]{2}{*}{$1 \mathrm{kHz}$} & OD & 4,98 & 1,66 & \multirow[t]{2}{*}{0,424} & Fem & 6,01 & 2,38 & \multirow[t]{2}{*}{0,483} \\
\hline & & OE & 5,24 & 2,62 & & Masc & 4,47 & 2,04 & \\
\hline & \multirow[t]{2}{*}{$1,5 \mathrm{kHz}$} & OD & 13,79 & 3,14 & \multirow[t]{2}{*}{0,480} & Fem & 14,98 & 5,19 & \multirow[t]{2}{*}{0,484} \\
\hline & & OE & 12,55 & 5,07 & & Masc & 11,76 & 3,51 & \\
\hline & \multirow[t]{2}{*}{$2 \mathrm{kHz}$} & OD & 16,96 & 3,27 & \multirow[t]{2}{*}{0,784} & Fem & 19,04 & 4,23 & \multirow[t]{2}{*}{0,264} \\
\hline & & OE & 17,09 & 4,61 & & Masc & 15,56 & 3,70 & \\
\hline & \multirow[t]{2}{*}{$3 \mathrm{kHz}$} & OD & 19,73 & 2,86 & \multirow[t]{2}{*}{0,583} & Fem & 21,18 & 3,93 & \multirow[t]{2}{*}{0,223} \\
\hline & & OE & 19,09 & 4,26 & & Masc & 18,06 & 3,33 & \\
\hline & $4 \mathrm{kHz}$ & OD & 18,69 & 3,53 & 0,814 & Fem & 20,62 & 3,75 & 0,139 \\
\hline & & OE & 18,68 & 3,46 & & Masc & 17,27 & 3,09 & \\
\hline & Geral & OD & 12,44 & 2,67 & 0,754 & Fem & 14,04 & 3,65 & 0,533 \\
\hline & & OE & 13,43 & 3,30 & & Masc & 12,19 & 2,52 & \\
\hline
\end{tabular}

Legenda: $\mathrm{EOAT}=$ emissões otoacústicas por transientes; $\mathrm{S} / \mathrm{R}=$ sinal/ruído; $\mathrm{OD}=$ orelha direita; $\mathrm{OE}=$ orelha esquerda; $\mathrm{IC}=$ intervalo de confiança; Fem = feminino; Masc = masculino

por estudo realizado em um hospital universitário na cidade de São Paulo.

Ao analisar a ocorrência de infecções congênitas na amostra de lactentes com risco auditivo observa-se uma ocorrência de $25,55 \%$ no presente estudo, também muito superior a outros estudos de encontraram ocorrência de $3 \%{ }^{(7)}, 4,3 \%{ }^{(12)}$, $10,93 \%{ }^{(13)}, 20 \%{ }^{(14)}$ e $4,3 \% \%^{(15)}$.

A sífilis foi a infecção congênita mais encontrada no grupo estudo (43\%), seguida do HIV (28\%). Dentre os fatores de risco que contribuem para a alta prevalência de sífilis e do HIV estão o baixo nível socioeconômico, a baixa escolaridade, exposição a riscos derivados de atividade sexual e, sobretudo, a falta de adequada assistência pré-natal ${ }^{(16,17)}$. Além da promiscuidade, atualmente, a precocidade da vida sexual tem tornado a sífilis a segunda mais grave doença sexualmente transmissível, perdendo somente para o $\mathrm{HIV}^{(18)}$. Outro fator relevante é a iniquidade no acesso aos serviços de saúde, para diagnóstico e tratamento precoces, das populações menos favorecidas socioeconomicamente, drasticamente evidenciada pelo crescimento persistente da proporção de óbitos por AIDS na categoria de raça/cor "preta" e "parda", em ambos os gêneros, entre 1998 e 2004.

A fim de caracterizar a função coclear em lactentes expostos à infecção congênita foram captadas as EOAT e EOAPD. Na captação das EOAT foi utilizado o estímulo clique, que avalia a cóclea somente até $4 \mathrm{kHz}$, no entanto é o mais sensível para perdas leves, assim como evidenciam outros estudos ${ }^{(7-9,19-25)}$. Na captação da EOAPD foi possível avaliar as altas frequências, com o custo de não ser sensível para perdas de grau leve assim como evidenciado em outras pesquisas ${ }^{(6,22,26-29)}$. Por esta razão, neste estudo optou-se por caracterizar os dois tipos de EOA.

Diversos estudos $^{(7,8,19,20,23,25)}$ apontam maior nível das EOAT na fase neonatal, no gênero feminino e para a orelha direita; os achados do presente estudo concordam com a literatura.

$\mathrm{O}$ efeito de grupo foi ligeiramente evidenciado no presente estudo sendo que tanto os níveis de resposta quanto relação $S / R$ das EOAT apresentaram valores maiores para o GC em relação ao GE com valores estatisticamente significantes apenas para o nível de resposta da banda de frequência de $1,5 \mathrm{kHz}$.

Novo estudo com o tamanho da amostra expandido seria necessário para verificar se esta tendência realmente é característica da população estudada, uma vez que não foram encontrados estudos na literatura analisando as EOAT na população neonatal com infecção congênita.

$\mathrm{Na}$ análise das EOAPD não foram verificadas diferenças estatisticamente significantes entre as orelhas tanto para o GC quanto para o GE, assim como em outros estudos ${ }^{(22,26-28)}$.

Não foram encontradas significantes diferenças entre os gêneros no GC. No grupo estudo o gênero feminino apresentou respostas maiores tanto para o nível de respostas quanto para 
Tabela 3. Nível de resposta e relação S/R das EOAT por grupo e banda de frequência

\begin{tabular}{|c|c|c|c|c|c|c|c|c|c|c|}
\hline EOAT & Frequência & Grupo & Média & Mediana & DP & Q1 & Q3 & $\mathrm{N}$ & IC & Valor de $p$ \\
\hline \multirow{12}{*}{$\begin{array}{l}\text { Nível de } \\
\text { resposta } \\
\text { (dB NPS) }\end{array}$} & \multirow[t]{2}{*}{$1 \mathrm{kHz}$} & $\mathrm{GC}$ & 13,41 & 13,00 & 5,08 & 10,55 & 17,45 & 44 & 1,50 & \multirow[t]{2}{*}{0,956} \\
\hline & & GE & 13,28 & 13,25 & 6,11 & 9,98 & 18,38 & 26 & 2,35 & \\
\hline & \multirow[t]{2}{*}{$1,5 \mathrm{kHz}$} & $\mathrm{GC}$ & 15,84 & 16,70 & 5,67 & 14,00 & 18,80 & 44 & 1,68 & \multirow[t]{2}{*}{0,050} \\
\hline & & GE & 14,13 & 14,35 & 5,15 & 12,08 & 17,00 & 26 & 1,98 & \\
\hline & \multirow[t]{2}{*}{$2 \mathrm{kHz}$} & $\mathrm{GC}$ & 18,35 & 18,75 & 4,55 & 15,43 & 20,53 & 44 & 1,35 & \multirow[t]{2}{*}{0,375} \\
\hline & & GE & 17,03 & 17,15 & 5,13 & 14,53 & 19,90 & 26 & 1,97 & \\
\hline & \multirow[t]{2}{*}{$3 \mathrm{kHz}$} & $\mathrm{GC}$ & 21,80 & 21,00 & 5,26 & 18,43 & 24,95 & 44 & 1,55 & \multirow[t]{2}{*}{0,236} \\
\hline & & GE & 19,74 & 20,20 & 5,40 & 15,63 & 23,35 & 26 & 2,08 & \\
\hline & \multirow[t]{2}{*}{$4 \mathrm{kHz}$} & $\mathrm{GC}$ & 18,80 & 18,20 & 5,94 & 14,93 & 22,80 & 44 & 1,76 & \multirow[t]{2}{*}{0,447} \\
\hline & & GE & 18,03 & 17,65 & 4,95 & 14,63 & 20,28 & 26 & 1,90 & \\
\hline & \multirow[t]{2}{*}{ Geral } & $\mathrm{GC}$ & 26,94 & 26,70 & 4,13 & 23,83 & 29,28 & 44 & 1,22 & \multirow[t]{2}{*}{0,145} \\
\hline & & GE & 25,39 & 25,45 & 4,13 & 23,70 & 27,75 & 26 & 1,59 & \\
\hline \multirow{12}{*}{$\begin{array}{l}\text { Relação S/R } \\
\text { (dB NPS) }\end{array}$} & \multirow[t]{2}{*}{$1 \mathrm{kHz}$} & $\mathrm{GC}$ & 6,82 & 5,05 & 7,56 & 0,00 & 9,28 & 44 & 2,23 & \multirow[t]{2}{*}{0,778} \\
\hline & & GE & 5,12 & 4,10 & 4,17 & 2,18 & 6,23 & 26 & 1,60 & \\
\hline & \multirow[t]{2}{*}{$1,5 \mathrm{kHz}$} & GC & 16,09 & 15,65 & 7,97 & 12,05 & 20,90 & 44 & 2,36 & \multirow[t]{2}{*}{0,129} \\
\hline & & GE & 13,12 & 11,50 & 8,06 & 6,18 & 17,98 & 26 & 3,10 & \\
\hline & \multirow[t]{2}{*}{$2 \mathrm{kHz}$} & $\mathrm{GC}$ & 19,03 & 19,65 & 6,06 & 14,48 & 23,80 & 44 & 1,79 & \multirow[t]{2}{*}{0,256} \\
\hline & & GE & 17,03 & 17,05 & 7,58 & 11,03 & 23,80 & 26 & 2,91 & \\
\hline & \multirow[t]{2}{*}{$3 \mathrm{kHz}$} & GC & 19,81 & 20,20 & 5,76 & 15,98 & 23,10 & 44 & 1,70 & \multirow[t]{2}{*}{0,747} \\
\hline & & GE & 19,38 & 19,60 & 6,90 & 14,28 & 23,13 & 26 & 2,65 & \\
\hline & \multirow[t]{2}{*}{$4 \mathrm{kHz}$} & $\mathrm{GC}$ & 16,29 & 15,75 & 6,16 & 11,85 & 19,80 & 44 & 1,82 & \multirow[t]{2}{*}{0,175} \\
\hline & & GE & 18,68 & 16,60 & 6,53 & 13,70 & 25,55 & 26 & 2,51 & \\
\hline & \multirow[t]{2}{*}{ Geral } & $\mathrm{GC}$ & 14,60 & 14,55 & 5,24 & 10,33 & 17,78 & 44 & 1,55 & \multirow[t]{2}{*}{0,236} \\
\hline & & GE & 12,97 & 11,30 & 5,69 & 8,63 & 16,45 & 26 & 2,19 & \\
\hline
\end{tabular}

Legenda: $\mathrm{EOAT}=$ emissões otoacústicas por transientes; $\mathrm{GC}=$ grupo comparação; $\mathrm{GE}=$ grupo estudo; $\mathrm{S} / \mathrm{R}=$ sinal/ruído; $\mathrm{OD}=$ orelha direita; $\mathrm{OE}=$ orelha esquerda; $\mathrm{DP}=$ desvio-padrão; $\mathrm{Q} 1$ = primeiro quartil; $\mathrm{Q} 3$ = terceiro quartil; $\mathrm{N}=$ número de sujeitos; $\mathrm{IC}$ = intervalo de confiança

a relação $\mathrm{S} / \mathrm{R}$ nas frequências de 1,5 e $2 \mathrm{kHz}$ com significância estatística apenas em $2 \mathrm{kHz}$ (p=0,023). Não foi possível estabelecer comparação com a literatura por essa variável não ter sido investigada.

O efeito de grupo nas EOAPD foi evidenciado no presente estudo, sendo que o GC apresentou maiores níveis de respostas e relação $\mathrm{S} / \mathrm{R}$ em relação ao $\mathrm{GE}$, em todas as frequências analisadas com significância estatística em $8 \mathrm{kHz}(\mathrm{p}=0,009)$. Outros estudos também verificaram menores respostas na EOAPD para o GE, tanto para RNPT com indicadores de risco auditivo ${ }^{(22)}$ quanto para RNPT expostos à medicação ototóxica ${ }^{(6)}$.

A análise dos dados entre os grupos (GC e GE) evidenciou que ambos os métodos EOAT e EOAPD são sensíveis para análise, entretanto através do método das EOAT foi possível verificar maior diferença de resposta entre os grupos, tanto no nível de resposta como na relação $S / R$.

Assim, a análise global dos resultados aponta para uma tendência de resposta reduzida de EOA na população de lactentes expostos a infecção congênita, confirmando a hipótese do presente estudo.

Sendo assim, torna-se importante a continuidade da investigação científica de lactentes expostos a infecções congênitas, principalmente com amostra ampliada, uma vez que esta população apresentou respostas diferenciadas de EOA.

Devido à grande variabilidade inter-sujeitos encontrada nas respostas das EOA, é necessário enfatizar que a resposta reduzida observada no GE, estatisticamente identificada como efeito de grupo, pode não ser detectada em casos individuais na prática clínica, e sim ratifica a recomendação do monitoramento do desenvolvimento auditivo destes lactentes.

\section{CONCLUSÃO}

A partir da análise crítica dos resultados, envolvendo o estudo das EOA de lactentes expostos a infecções congênitas, foi possível observar menores níveis de resposta das EOAT do que o grupo comparação com significância estatística para a banda de frequência de $1,5 \mathrm{kHz}$, e das EOAPD principalmente nas frequências agudas, com significância estatística para $8 \mathrm{kHz}$.

\section{AGRADECIMENTOS}

À Fundação de Amparo à Pesquisa do Estado de São Paulo (FAPESP), pelo apoio fornecido em forma de bolsa de iniciação científica (processo $\mathrm{n}^{\circ} 06 / 55140-8$ ). 
Tabela 4. Nível de resposta e relação S/R das EOAPD segundo o grupo por frequência

\begin{tabular}{|c|c|c|c|c|c|c|c|c|c|c|}
\hline EOAPD & Frequência & Grupo & Média & Mediana & DP & Q1 & Q3 & $\mathrm{N}$ & IC & Valor de $p$ \\
\hline \multirow{12}{*}{$\begin{array}{l}\text { Nível de } \\
\text { resposta } \\
\text { (dB NPS) }\end{array}$} & \multirow[t]{2}{*}{$1,5 \mathrm{kHz}$} & GC & 15,40 & 15,60 & 7,68 & 11,48 & 19,68 & 32 & 2,66 & \multirow[t]{2}{*}{0,785} \\
\hline & & GE & 15,91 & 15,75 & 5,50 & 12,55 & 20,30 & 24 & 2,20 & \\
\hline & \multirow[t]{2}{*}{$2 \mathrm{kHz}$} & GC & 15,32 & 16,35 & 5,97 & 10,55 & 19,13 & 32 & 2,07 & \multirow[t]{2}{*}{0,849} \\
\hline & & GE & 15,33 & 16,40 & 6,44 & 12,08 & 19,30 & 24 & 2,58 & \\
\hline & \multirow[t]{2}{*}{$3 \mathrm{kHz}$} & GC & 16,53 & 17,40 & 5,38 & 13,98 & 20,55 & 32 & 1,86 & \multirow[t]{2}{*}{0,205} \\
\hline & & GE & 14,41 & 15,25 & 6,22 & 10,38 & 18,63 & 24 & 2,49 & \\
\hline & \multirow[t]{2}{*}{$4 \mathrm{kHz}$} & GC & 16,55 & 18,00 & 7,31 & 10,93 & 22,10 & 32 & 2,53 & \multirow[t]{2}{*}{0,471} \\
\hline & & GE & 16,38 & 16,15 & 5,23 & 13,50 & 20,03 & 24 & 2,09 & \\
\hline & \multirow[t]{2}{*}{$6 \mathrm{kHz}$} & GC & 16,99 & 19,75 & 7,62 & 9,73 & 21,50 & 32 & 2,64 & \multirow[t]{2}{*}{0,172} \\
\hline & & GE & 15,66 & 16,15 & 5,85 & 12,98 & 18,40 & 24 & 2,34 & \\
\hline & \multirow[t]{2}{*}{8 kHz } & GC & 24,39 & 28,85 & 9,91 & 19,35 & 31,08 & 32 & 3,43 & \multirow[t]{2}{*}{$0,009^{*}$} \\
\hline & & GE & 19,62 & 19,85 & 6,83 & 16,75 & 23,83 & 24 & 2,73 & \\
\hline \multirow{12}{*}{$\begin{array}{l}\text { Relação S/R } \\
\text { (dB NPS) }\end{array}$} & \multirow[t]{2}{*}{$1,5 \mathrm{kHz}$} & GC & 8,84 & 7,00 & 5,88 & 6,18 & 12,03 & 32 & 2,04 & \multirow[t]{2}{*}{0,934} \\
\hline & & GE & 7,83 & 7,10 & 3,25 & 6,38 & 9,90 & 24 & 1,30 & \\
\hline & \multirow[t]{2}{*}{$2 \mathrm{kHz}$} & GC & 11,90 & 10,20 & 6,20 & 7,08 & 17,00 & 32 & 2,15 & \multirow[t]{2}{*}{0,888} \\
\hline & & GE & 12,27 & 9,45 & 6,80 & 7,40 & 17,30 & 24 & 2,72 & \\
\hline & \multirow[t]{2}{*}{$3 \mathrm{kHz}$} & GC & 15,40 & 16,45 & 6,88 & 8,63 & 20,68 & 32 & 2,39 & \multirow[t]{2}{*}{0,614} \\
\hline & & GE & 14,13 & 12,70 & 8,10 & 8,68 & 20,73 & 24 & 3,24 & \\
\hline & \multirow[t]{2}{*}{$4 \mathrm{kHz}$} & GC & 19,21 & 18,65 & 8,64 & 12,35 & 25,40 & 32 & 2,99 & \multirow[t]{2}{*}{0,208} \\
\hline & & GE & 16,34 & 16,85 & 8,03 & 10,93 & 19,93 & 24 & 3,21 & \\
\hline & \multirow[t]{2}{*}{$6 \mathrm{kHz}$} & GC & 18,85 & 18,75 & 8,29 & 11,48 & 26,03 & 32 & 2,87 & \multirow[t]{2}{*}{0,116} \\
\hline & & GE & 15,75 & 16,70 & 7,46 & 9,55 & 21,73 & 24 & 2,98 & \\
\hline & \multirow[t]{2}{*}{$8 \mathrm{kHz}$} & GC & 18,34 & 19,50 & 9,79 & 9,98 & 27,10 & 32 & 3,39 & \multirow[t]{2}{*}{0,183} \\
\hline & & GE & 15,09 & 13,70 & 6,74 & 9,20 & 20,20 & 24 & 2,70 & \\
\hline
\end{tabular}

* Valores estatisticamente significantes $(p<0,05)$

Legenda: $\mathrm{EOAPD}=$ emissões otoacústicas produto de distorção; $\mathrm{GC}=$ grupo comparação; $\mathrm{GE}=$ grupo estudo; $\mathrm{S} / \mathrm{R}=$ sinal/ruído; $\mathrm{OD}=$ orelha direita; $\mathrm{OE}=$ orelha esquerda; DP = desvio-padrão; Q1 = primeiro quartil; Q3 = terceiro quartil; N = número de sujeitos; IC = intervalo de confiança

\begin{abstract}
Purpose: To analyze otoacoustic emissions (OAE) in infants exposed to intrauterine infection. Methods: Transient OAE (TEOAE) and distortion product OAE (DPOAE) were performed in 40 infants: 14 with and/or exposed to some type of congenital infection (Study Group) and 26 without exposition and with no risk factors for hearing loss (Control Group). Inclusion criteria were: Control Group: healthy full-term infants, with no risk factors for hearing loss; Study Group: full-term infants or preterms with corrected gestational age between 37 and 41 weeks at the date of assessment, exposed to some type of intrauterine infection or with congenital infection. The statistical analysis of the data set was performed using the non-parametric tests of Wilcoxon and Mann-Whitney, and the technique of confidence interval for the mean. Results: Lower levels of response and signal/noise ratio in TEOAE and DPOAE were observed in the Study Group, when compared to the Control Group, with significant statistical values for DPOAE in $8 \mathrm{kHz}$, and for TEOAE in $1.5 \mathrm{kHz}$. Conclusion: The exposure to intrauterine infections might attenuate the response level in OAE during the neonatal period.
\end{abstract}

Keywords: Otoacoustic emissions, spontaneous; Infant; Fetal diseases; Infection/congenital; Risk factors

\title{
REFERÊNCIAS
}

1. Lewis DR. As habilidades auditivas do recém-nascido e a triagem auditiva neonatal. In: Andrade CRF. Fonoaudiologia em berçário normal e de risco. São Paulo: Lovise; 1996.p.149-68.
2. Northern JL, Downs MP. Audição na infância 9a ed. Rio de Janeiro: Guanabara Koogan; 2002.

3. Botelho DL. Alterações auditivas por moléstia infecto-contagiosa: 
análise de um programa de detecção precoce [dissertação]. São Paulo: Faculdade de Fonoaudiologia da Pontifícia Universidade Católica de São Paulo; 2002.

4. Leone ADC. Programa de triagem auditiva neonatal: caracterização dos neonatos do berçário de alto risco da Irmandade da Santa Casa de São Paulo [trabalho de conclusão de curso]. São Paulo: Faculdade de Ciências Médicas da Santa Casa de São Paulo; 2005.

5. Kemp DT. Stimulated acoustic emissions from within the human auditory system. J Acoust Soc Am. 1978;64(5):1386-92.

6. Ruggieri-Marone M, Schochat E. Distortion product otoacoustic emissions in newborns treated by ototoxic drugs. Rev Laryngol Otol Rhinol (Bord). 2007;128(1-2):41-6.

7. Durante AS, Carvallo RM. Contralateral suppression of linear and nonlinear transient evoked otoacoustic emissions in neonates at risk for hearing loss. J Commun Disord. 2008;41(1):70-83.

8. Bassetto MC, Chiari BM, Azevedo MF. Emissões otoacústicas evocadas transientes (EOAT): amplitude da resposta em recém-nascidos a termo e pré-termo. Rev Bras Ororrinolaringol. 2003;69(1):84-92.

9. Chabert R, Guitton MJ, Amram D, Uziel A, Pujol R, Lallemant JG, Puel JL. Early maturation of evoked otoacoustic emissions and medial olivocochlear reflex in preterm neonates. Pediatr Res. 2006;59(2):305-8.

10. Diniz EMA, Ramos JLA, Vaz FAC. Infecções congênitas e perinatais. In: Marcondes E, Vaz FAC, Ramos JLA. Pediatria básica. São Paulo: Sarvier; 2003. p. 523-53.

11. Torrico P, Gómez C, López- Rios J, Cáceres MC,Trinidad G, Serrano M. Influencia de la edad en las otoemisiones acústicas para el screening de hipoacusia infantil. Acta Otorrinolaringol Esp. 2004;55(4):153-9.

12. Vieira EP, Miranda EC, Azevedo MF, Garcia MV. Ocorrência dos indicadores de risco para a deficiência auditiva infantil no decorrer de quatro anos em um programa de triagem auditiva neonatal de um hospital público. Rev Soc Bras Fonoaudiol. 2007;12(3):214-20.

13. Torrico Román P, Trinidad Ramos G, Cáceres Morillo MC, Lozano Sánchez S, López-Ríos Velasco J. Detección precoz de hipoacusias en recién nacidos mediante otoemisiones acústicas con Echocheck. An Pediatr. 2001;54(3):283-9.

14. Iñíguez RC, Cevo TE, Fernández FL, Godoy CB, Iñíguez SR. Detección precoz de pérdida auditiva en niños con factores de riesgo. Rev Otorrinolaringol Cir Cabeza Cuello. 2004;64(2):99-104.

15. Srisuparp P, Gleebbur R, Ngerncham S, Chonpracha J, Singkampong J. High- risk neonatal hearing screening program using automated screening device performed by trained nursing personnel at Siriraj
Hospital: yield and feasibility. J Med Assoc Thai. 2005; 88(Suppl 8):S176-82

16. De Lorenzi DRS, Madi JM. Sífilis congênita como indicador de assistência pré-natal. Rev Bras Ginecol Obstet. 2001;23(10):647-52.

17. Santos NJS, Tayra A, Silva SR, Buchalla CM, Laurenti R. A aids no Estado de São Paulo. As mudanças no perfil da epidemia e perspectivas da vigilância epidemiológica. Rev Bras Epidemiol. 2002;5(3):286-310.

18. Brock R, Martinez SMRC. Infecções congênitas. In: Bassetto MCA, Brock R, Wajnsztejn R. Neonatologia: um convite à atuação fonoaudiológica. São Paulo: Lovise; 1998. p. 171-9.

19. Aidan D, Lestang P, Avan P, Bonfils P. Characteristics of transientevoked otoacoustic emissions (TEOES) in neonates. Acta Otolaryngol. 1997;117(1):25-30

20. Newmark M, Merlob P, Bresloff I, Olsha M, Attias J. Click evoked otoacoustic emissions: inter-aural and gender differences in newborns. J Basic Clin Physiol Pharmacol. 1997;8(3):134-9.

21. Paludetti G, Ottaviani F, Fetoni AR, Zuppa AA, Tortorolo G. Transient evoked otoacoustic emissions (TEOAEs) in new-borns: normative data. Int J Pediatr Otorhinolaryngol. 1999;47(3):235-41.

22. Cerruti VQ. Estudo das emissões otoacústicas evocadas em neonatos: transiente e produto de distorção [tese]. [São Paulo(SP)]: Instituto de Psicologia, Universidade de São Paulo; 2000.

23. Durante AS, Carvallo RM. Contralateral suppression of otoacoustic emissions in neonates. Int J Audiol. 2002;41(4):211-5.

24. Fuzetti C, Lewis DR. Emissões otoacústicas espontâneas e evocadas por estímulo transiente em recém-nascidos. Pró-Fono. 2003;15(2):189-98.

25. Durante AS, Carvallo RMM, Costa FS, Soares JC. Características das emissões otoacústicas por transientes em programa de triagem auditiva neonatal. Pró- Fono. 2005;17(2):133-40.

26. Vallejo JC, Soares E, Chiriboga LM. Análise do padrão de respostas em neonatos normais para emissões otoacústicas evocadas por produto de distorção. Rev Bras Otorrinolaringol. 1998;64(3):251-4.

27. Raineri GG, Coube CZV, Costa Filho OA, Alvarenga KF. Emissões otoacústicas evocadas - produto de distorção em neonatos audiologicamente normais. Rev Bras Otorrinolaringol. 2001;67(5):1-9.

28. Pinto VS, Lewis DR. Emissões otoacústicas: produto de distorção em lactentes até dois meses de idade. Pró-Fono. 2007;19(2):195-204.

29. Zang Z, Jiang ZD. Distorcion product otoacoustic emissions during the first year in term infants: a longitudinal study. Brain Dev. 2007;29(6):346-51. 